\title{
Vibrotaktile Codierung von Standardfunktionen einer Menüführung
}

\author{
Tobias Stein ${ }^{1} \cdot$ Ludger Schmidt $^{1}$
}

Online publiziert: 3. März 2020

(c) Der/die Autor(en) 2020

\section{Zusammenfassung}

Tactons sind taktile Nachrichten, die dem Nutzer Informationen über den haptischen Sinneskanal übermitteln, und können beispielsweise bei der Interaktion mit Touchscreens von Nutzen sein. In dieser Studie wird die systematische Erarbeitung eines Tacton-Sets für Standardfunktionen einer Menüführung (im Einzelnen die Funktionen Beenden, Bestätigen, Hauptmenü, Hilfe, Korrektur, Lauter, Leiser sowie Zurück) vorgestellt. Neben existierenden Erkenntnissen aus der Literatur wurden bei der Gestaltung des Tacton-Sets ebenfalls die Ergebnisse einer Gruppenbefragung berücksichtigt, in der die Teilnehmer Ideen zur konkreten Ausgestaltung der Tactons äußern konnten. Das zunächst entstandene Tacton-Set wurde unter drei standardisierten Versuchsbedingungen von 54 Probanden evaluiert und lieferte eine Gesamterkennungsrate von $0,70 \pm 0,19$. Die Ergebnisse der einzelnen Tactons wurden analysiert und Verbesserungen hinsichtlich deren Gestaltung umgesetzt. Anschließend wurde das weiterentwickelte Tacton-Set unter den gleichen Versuchsbedingungen wie zuvor erneut

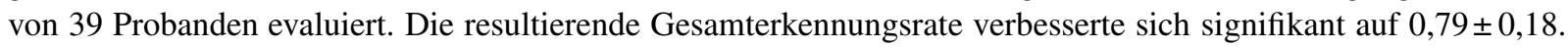

Praktische Relevanz In alltäglichen Umgebungen mit zunehmender Informationsdichte wird die Übermittlung von Informationen über den haptischen Sinneskanal immer wichtiger. Diese Studie beinhaltet wichtige Erkenntnisse für die Gestaltung und schrittweise Verbesserung von Tactons im Allgemeinen und liefert einen Vorschlag für die konkrete Umsetzung eines Tacton-Sets für genannte Standardfunktionen. Es wird zudem deutlich, dass weitere Forschung nötig ist, um den Einsatz von Tactons zu etablieren.

Schlüsselwörter Vibrotaktile Information $\cdot$ Codierung $\cdot$ Tactons $\cdot$ Haptik $\cdot$ Mensch-Maschine-Interaktion

\section{Vibrotactile coding of standard functions in application menues}

\begin{abstract}
Tactons are structured tactile messages for information transmission to the user via the tactile sense. This study presents a systematic development of a tacton set for standard functions in computer application menus (i.e. close, OK/confirm, main menu, help, delete, volume up, volume down and back). Hereby, existing findings of other researchers as well as the results of a group work conducted to collect subjective ideas for tacton design were considered. 54 participants evaluated the initially designed tacton set under various conditions. The overall recognition rate was $0.70 \pm 0.19$. Afterwards, the results of the single tactons were analyzed and improvements concerning their design were implemented. This advanced set of tactons was evaluated again under the same conditions by 39 participants. The overall recognition rate improved significantly to $0.79 \pm 0.18$.

Practical Relevance Due to the increasing information density in daily life, the transmission of information via the tactile sense becomes more and more important. This study contains important findings of design and gradual optimization of tactons in general and provides a recommendation of a tacton set for standard functions in computer application menus. To establish the use of tactons in daily life, more research in this field is needed.
\end{abstract}

Keywords Vibrotactile information $\cdot$ Coding $\cdot$ Tactons $\cdot$ Haptics $\cdot$ Human-machine interaction

Univ.-Prof. Dr.-Ing. Ludger Schmidt

L.Schmidt@uni-kassel.de
1 Fachgebiet Mensch-Maschine-Systemtechnik, Universität Kassel, Mönchebergstraße 7, 34125 Kassel, Deutschland 


\section{Hintergrund und Ziel}

Tactons sind Vibrationsmuster, die es ermöglichen, über den haptischen Sinneskanal Informationen zu übermitteln. Dies ist durch die Modifikation der einzelnen Parameter von Vibrationen (Frequenz, Amplitude, Dauer, Rhythmus etc.) möglich. Sie sind nützlich, wenn andere Sinneskanäle bereits beansprucht werden oder aufgrund der Umgebungsbedingungen nicht zur Verfügung stehen (Freeman et al. 2017; Brewster und Brown 2004), beispielsweise aufgrund von Blickabwendung oder lauten Umgebungsgeräuschen. Tactons können dabei verschiedene abstrakte Bedeutungen wie Befehle, Zustände oder Warnungen darstellen, sofern die Benutzer geschult sind, sie zu erkennen und korrekt zu interpretieren (Luzhnica und Veas 2019). Nach wie vor ist grundlegende Forschung nötig, um zu verstehen, wie solche Tactons am besten zu gestalten sind (Barber und Beck 2015). Bereits bekannt ist, dass einzelne Parameter besser geeignet sind, um Informationen zu codieren als andere. So lieferten beispielsweise die Parameter Rhythmus und Rauheit - ein Parameter, der generiert wird, wenn eine Sinusschwingung mit einer zweiten, unterschiedlichen Sinusschwingung moduliert wird (Brown et al. 2005) vielversprechende Ergebnisse (Brown et al. 2005, 2006; Constantin und Brewster 2010), und auch die Frequenz ist geeignet, wohingegen die Kurvenform weniger geeignet scheint (Stein et al. 2017). Zudem wurden bereits Untersuchungen angestellt, die aufzeigen, welche Parameter ähnlich bzw. unterschiedlich wahrgenommen werden (z. B. Jones und Singhal 2018), und wie sich einzelne Parameter auf die wahrgenommene Ausprägung von Daten beziehen, die in realen Kontexten genutzt werden können, beispielsweise Distanz, Fehler und Gefahr (Ferguson et al. 2018).

Bei der Realisierung gilt es außerdem, die Wahrnehmungsschwellen des Menschen zu berücksichtigen. Die absolute vibrotaktile Wahrnehmungsschwelle des Menschen beschreibt die geringste Intensität eines Reizes, die eine gerade noch wahrnehmbare Empfindung auslöst, wohingegen die Unterschiedsschwelle charakterisiert ist als die kleinste Änderung der Reizstärke, die gerade noch als Veränderung der Intensität empfunden wird (Behrends et al. 2012). Die Unterschiedsschwelle ist dabei abhängig von der Intensität des Ausgangsreizes (Weber-Fechner-Gesetz).

Tactons wurden bereits in verschiedenen Untersuchungen erfolgreich eingesetzt, beispielsweise um die Beendigung eines Downloads zu signalisieren (Brewster und King 2005), Art und Dringlichkeit einer eingehenden Nachricht zu codieren (Brown et al. 2005), über Art, Wichtigkeit und verbleibende Zeit bis zu einem anstehenden Termin zu informieren (Brown et al. 2006) oder Informationen über die Geschehnisse in der Umgebung bereitzustellen (Constantin und Brewster 2010). Die Ergebnisse dieser Studien zeigen, dass Tactons durchaus genutzt werden können, um
Informationen über den haptischen Sinneskanal zu transportieren, und dabei gute Ergebnisse erzielt werden können. Bei der Nutzung von räumlich-zeitlichen Vibrationsmustern zur Codierung aller 26 Buchstaben des Alphabets konnten Luzhnica et al. (2016) nach mehreren Stunden Training Erkennungsraten von $90 \%$ und mehr erzielen. Ebenso gute Ergebnisse wurden in einem zweiten Experiment mit 10 Buchstaben erreicht, in dem parallel eine weitere Aufgabe zur Ablenkung erfüllt werden musste (Luzhnica und Veas 2019).

Um dieses Potenzial im alltäglichen Leben nutzen zu können, ist eine Standardisierung sinnvoll. Gerade bei der Interaktion mit Touchscreens, bei der eine haptische Rückmeldung fehlt, da mechanische Tasten durch eine glatte, feste Oberfläche ersetzt wurden, scheinen Tactons eine vielversprechende Kompensationsmöglichkeit zu sein (MacLean et al. 2017). Vor allem für häufig vorkommende Funktionen auf verschiedenen Geräten wie beispielsweise Automaten, Computern und mobilen Endgeräten wie Smartphone und Tablet-PC, ist ein standardisiertes Tacton-Set sinnvoll, da die Bedeutung der einzelnen Tactons dann nicht immer wieder neu erlernt werden muss, sondern nach einiger Zeit von den Nutzern verinnerlicht sein wird. Tactons können hierbei einen erheblichen Vorteil bieten, wenn diese dem Nutzer nicht nur die Ausführung quittieren, sondern zusätzlich vorher schon vermitteln, auf welchem Eingabeelement des Bildschirms sich sein Finger befindet und welche Funktion hier hinterlegt ist, um möglichen Fehleingaben vorzubeugen. Zudem kann so der visuelle und/oder auditive Sinneskanal entlastet und für andere Aufgaben genutzt werden (z. B. Bedienung des Infotainment-Systems im Auto bei gleichzeitigem Blick auf die Straße). In der vorliegenden Studie wird das entworfene Tacton-Set zunächst unter Laborbedingungen hinsichtlich grundsätzlicher Machbarkeit evaluiert. In einem nächsten Schritt soll eine Evaluation im Feld mit konkreten Anwendungsszenarien erfolgen.

Grundsätzlich muss sichergestellt sein, dass sich die Tactons untereinander sehr gut voneinander unterscheiden, da diese nicht nur relativ miteinander verglichen werden sollen, sondern eine absolute Identifikation ermöglicht werden muss, was Nutzern erheblich schwerer fällt (Brewster und Brown 2004). Des Weiteren ist eine möglichst einfache und schnelle Erlernbarkeit wünschenswert, was bei einem derart abstrakten und vergleichsweise unbekannten Konstrukt wie einem Tacton schwierig zu verwirklichen ist. Ein vielversprechender Ansatz ist hierbei, die Tactons so zu gestalten, dass die Identifizierung der von ihnen codierten Funktionen auf eine intuitive Weise erfolgen kann.

Chan et al. $(2005,2008)$ nutzten beispielsweise Metaphern, um die Erlernbarkeit von den in ihrer Studie genutzten, haptisch codierten Funktionen zu erleichtern. Bei der Gestaltung der Tactons griffen sie auf bekannte Geräusche und Tätigkeiten zurück, die mit den in ihrer Studie codier- 
ten Funktionen in Verbindung gebracht und demnach für die haptische Codierung dieser Funktionen genutzt wurden. Die sieben Tactons der codierten Funktionen mussten daher von den Versuchspersonen vermutlich nicht erst von Grund auf erlernt, sondern konnten nach einer Erklärung der Bedeutung und einer kurzen Eingewöhnungsphase schnell korrekt zugeordnet werden. Dafür spricht ebenfalls die hohe Gesamterkennungsrate des Versuchs von 95,7\% (Chan et al. 2005, 2008).

\section{Gruppenbefragung}

Um ein Tacton-Set für Standardfunktionen gestalten zu können, wurden zur Vorbereitung der hier vorgestellten Studie durch eine Gruppenbefragung potenzieller Nutzer Ideen zur Ausgestaltung verschiedener Standardfunktionen mittels Vibrationsmustern sowie deren Konnotation gesammelt. Im Einzelnen wurden die Funktionen Beenden, Bestätigen, Hauptmenü, Hilfe, Korrektur, Lauter, Leiser sowie Zurück betrachtet, welche in Menüführungen verschiedener Anwendungen (Bankterminal, Fahrkartenautomat, Infotainment-Systeme etc.) besonders häufig vorkommen.

Zehn (sechs männlich, vier weiblich) Teilnehmer im Alter von 38,4 $\pm 16,2$ Jahren (Spannweite 25-69 Jahre) nahmen an dieser Befragung teil. Fünf der Teilnehmer waren Studierende, drei berufstätig und zwei im Ruhestand. Somit wurde das Ziel erreicht, eine möglichst heterogene Gruppe zusammenzustellen, um unterschiedliche Ansätze und vielfältige Ideen zusammenzutragen (Franke 2016).

Die Teilnehmer wurden vom Leiter der Gruppenbefragung durch eine Präsentation zunächst umfassend in die Thematik der Tactons sowie deren mögliche modifizierbare Parameter eingeführt und ihre Aufgaben sowie die Zielsetzung der Befragung definiert. Anschließend wurden die Ideen mit Methoden des Brainstormings und Brainwritings zusammengetragen. Während beim Brainstorming solche Ideen mündlich geäußert werden, so werden diese beim Brainwriting schriftlich festgehalten. Dies ermöglicht es ebenfalls zurückhaltenden oder unsicheren Personen, ihre Ideen - auch in anonymer Form - einzubringen (Schmid 1992). Beide Methoden haben gemeinsam, dass möglichst viele Ideen sowie auch ungewöhnliche Vorschläge erwünscht sind (Franke 2016) - eine Bewertung der gesammelten Beiträge erfolgt erst im Nachgang.

Die Teilnehmer sollten nun in Kleingruppen (drei bis vier Personen) einzelne aufgebaute Stationen durchlaufen, wobei eine Station eine der betrachteten Standardfunktionen repräsentierte (pro Station etwa fünf Minuten Zeit). An jeder Station standen den Teilnehmern sowohl Stifte und Plakate als auch Aufnahmegeräte für Ton und Video zur Verfügung, um ihre Ideen zu äußern. Die Ergebnisse der vorherigen Kleingruppen konnten hierbei aufgegrif- fen und weiterentwickelt werden. Die Gruppenbefragung schloss mit einer gemeinsamen Abschlussdiskussion aller Teilnehmer, bei der Hintergründe zu einzelnen Ideen detailliert erfragt und Ergänzungen zu geäußerten Vorschlägen vorgenommen werden konnten.

Insgesamt wurden 181 verschiedene Ideen gesammelt, wobei 122 Vorschläge auf die Poster geschrieben sowie 54 Tonaufnahmen und fünf Videoaufnahmen angefertigt wurden. Hervorzuheben ist an dieser Stelle, dass trotz der verhältnismäßig kurzen Auseinandersetzung mit der für alle Teilnehmer unbekannten und abstrakten Thematik der Tactons bereits einige Antworten bezüglich einer konkreten Umsetzung eine hohe Qualität aufwiesen, z. B. „Die Funktionen Lauter und Leiser könnte man über die Amplitude/ Frequenz darstellen“ oder „Die Funktion Bestätigen sollte ohne Rauheit realisiert werden“.

Zur Auswertung wurden diese Ergebnisse zunächst transkribiert. Anschließend wurden die Ergebnisse thematisch verglichen und ähnliche Beiträge $\mathrm{zu}$ übergeordneten Themeneinheiten gruppiert (Meuser und Nagel 2009). Im letzten Schritt erfolgte eine Selektion der Daten, wobei bestimmte Vorschläge aussortiert wurden - einerseits solche Ideen, die nicht zur definierten Fragestellung passten, und andererseits solche, die kaum genannt wurden oder wenig Unterstützung von anderen Gruppenmitgliedern bekamen (Ullrich 2006). Die hiernach vorliegenden Ergebnisse dieser Befragung werden aufgegriffen und bei der Gestaltung der einzelnen Funktionen berücksichtigt (siehe Abschn. 3.1 und 4.1).

Die Ideensammlung dieser heterogenen Teilnehmergruppe war ein wichtger Bestandteil für die anschließende Ausgestaltung des Tacton-Sets für Standardfunktionen, da nun sowohl geeignete Parameter, welche aus der Literatur bekannt sind (z. B. Brewster und Brown 2004; Brown et al. 2006; Constantin und Brewster 2010; Stein et al. 2017; van Erp 2002), als auch Vorschläge zur konkreten Gestaltung des Tacton-Sets berücksichtigt und umgesetzt werden konnten.

\section{Entwurf des Tacton-Sets für Standardfunktionen}

Neben der Berücksichtigung der Ergebnisse der Gruppenbefragung und der bereits vorhandenen Erkenntnisse aus der Literatur wird zudem versucht, die Tactons für die genannten Standardfunktionen möglichst intuitiv zu gestalten. Damit liefert diese Studie einen ersten Vorschlag eines solchen Tacton-Sets, welches im Folgenden beschrieben und unter verschiedenen Versuchsbedingungen evaluiert wird. 


\subsection{Codierung der Standardfunktionen}

Die Codierung der genannten Standardfunktionen wurde folgendermaßen umgesetzt:

Beenden wurde durch eine $500 \mathrm{~ms}$ andauernde, raue Vibration $(250 \mathrm{~Hz}$ Sinusfrequenz moduliert mit einer $50 \mathrm{~Hz}$ Sinusfrequenz, siehe Brown et al. (2005)) codiert. Durch die genutzte Rauheit wurden die meist negativen Konnotationen der Versuchspersonen aus der Gruppenbefragung aufgegriffen.

Die Funktion Bestätigen wurde durch eine kurze $(100 \mathrm{~ms})$ und eine lange $(400 \mathrm{~ms})$ Vibration, mit einer Pause von 80 ms dazwischen, codiert. Die positive Konnotation dieser Funktion - einvernehmlich geäußert in der Gruppenbefragung - wurde durch die angenehme Frequenz von $150 \mathrm{~Hz}$ (Stein et al. 2016) aufgegriffen. Zudem sollte durch die Länge der einzelnen Vibrationen die Aussprache der beiden Silben des Wortes „O_kay“ nachempfunden werden.

Die Funktion Hauptmenii wurde durch zwei kurze (je $150 \mathrm{~ms}$ mit $100 \mathrm{~ms}$ Pause), angenehme Vibrationen $-150 \mathrm{~Hz}$ wurden als Frequenz gewählt (Stein et al. 2016) - dargestellt. Somit werden neben der Annehmlichkeit dieses Vibrationsmusters ebenso die Vorschläge zu einer einfachen, kurzen und prägnanten Codierung dieser Funktion aus der Gruppenbefragung erfüllt. Bei nahezu gleicher Dauer (Hauptmenü: insgesamt $400 \mathrm{~ms}$ ) wie die Funktion Beenden unterscheiden sich diese Vibrationsmuster in Frequenz und Rauheit, die beiden Parameter, welche die beste Unterscheidbarkeit ergaben (Stein et al. 2017), sowie der Anzahl ihrer Einzelimpulse.

Für die Codierung der $\boldsymbol{H i l f e}$-Funktion wurde der bekannte Morse-Code genutzt, welcher auch in der Gruppenbefragung häufig genannt wurde. Drei kurze, drei lange (je $80 \mathrm{~ms}$ bzw. $200 \mathrm{~ms}$ mit jeweils $80 \mathrm{~ms}$ Pause dazwischen) und abermals drei kurze Vibrationen codierten diese Funktion, wodurch sie die längste Dauer aufwies (1800 ms). Mit einer Frequenz von $250 \mathrm{~Hz}$ ist diese Funktion besonders gut wahrnehmbar (Verrillo 1992; Gescheider et al. 2004).

Korrektur wurde durch drei Vibrationen (je $200 \mathrm{~ms}$ lang mit jeweils $50 \mathrm{~ms}$ Pause dazwischen) codiert, welche durch die genutzte Rauheit $(250 \mathrm{~Hz}$ Sinusschwingung moduliert mit $30 \mathrm{~Hz}$ Sinusschwingung, siehe (Brown et al. 2005)) die durchweg negative Konnotation aus der Gruppenbefragung aufgreifen. Somit wurde eine andere Rauheit als in der bereits beschriebenen Funktion Beenden genutzt. Des Weiteren unterscheiden sich diese beiden Funktionen bei vergleichbarer Dauer (Korrektur: insgesamt $700 \mathrm{~ms}$ ) durch die Anzahl ihrer Einzelimpulse.

Die Funktion Lauter wurde durch drei ohne Pause aufeinander folgenden und in ihrer Intensität zunehmenden Vibrationen von je $150 \mathrm{~ms}$ Länge codiert. Hierbei wurde wie bei der Hilfe-Funktion eine Frequenz von $250 \mathrm{~Hz}$ gewählt, um eine gute Wahrnehmbarkeit sicherzustellen (Verrillo 1992; Gescheider et al. 2004). Durch den großen Unterschied in der Dauer (Lauter: insgesamt $450 \mathrm{~ms}$ ) sollte die Unterscheidbarkeit zwischen diesen beiden Funktionen gegeben sein.

Leiser wurde analog zur Funktion Lauter gestaltet, wobei der einzige Unterschied darin bestand, dass die Intensität der drei Vibrationen abnahm. Somit wurde die Idee aus der Gruppenbefragung umgesetzt, die Funktionen Lauter und Leiser über die Amplitude zu codieren.

Die Zurück-Funktion wurde durch vier Vibrationen mit je $100 \mathrm{~ms}$ Dauer codiert, die in ihrer Intensität zunahmen, einer Pause von $100 \mathrm{~ms}$ und erneuten vier Vibrationen, die in ihrer Intensität abnahmen. Somit wurde ein Vibrationsmuster zunächst „,vorwärts“ und dann „rückwärts“ abgespielt, was die Funktion Zurück anschaulich und intuitiv darstellen sollte. Dabei wurde eine raue Vibration gewählt $(250 \mathrm{~Hz}$ Sinusfrequenz moduliert mit einer $50 \mathrm{~Hz}$ Sinusfrequenz, siehe (Brown et al. 2005)), um die in der Gruppenbefragung genannte negative Konnotation aufzugreifen. Die Funktion Beenden, welche ebenfalls diese Rauheit nutzte, unterscheidet sich sowohl in der Anzahl der Einzelimpulse als auch der Dauer (Zurück: insgesamt $900 \mathrm{~ms}$ ), um eine Unterscheidbarkeit dieser beiden Funktionen zu gewährleisten.
Abb. 1 Stationäres Touchscreen-Mockup (a) und dessen technische Komponenten (b) Fig. 1 Stationary touch screen mock-up (a) and its technological components (b)
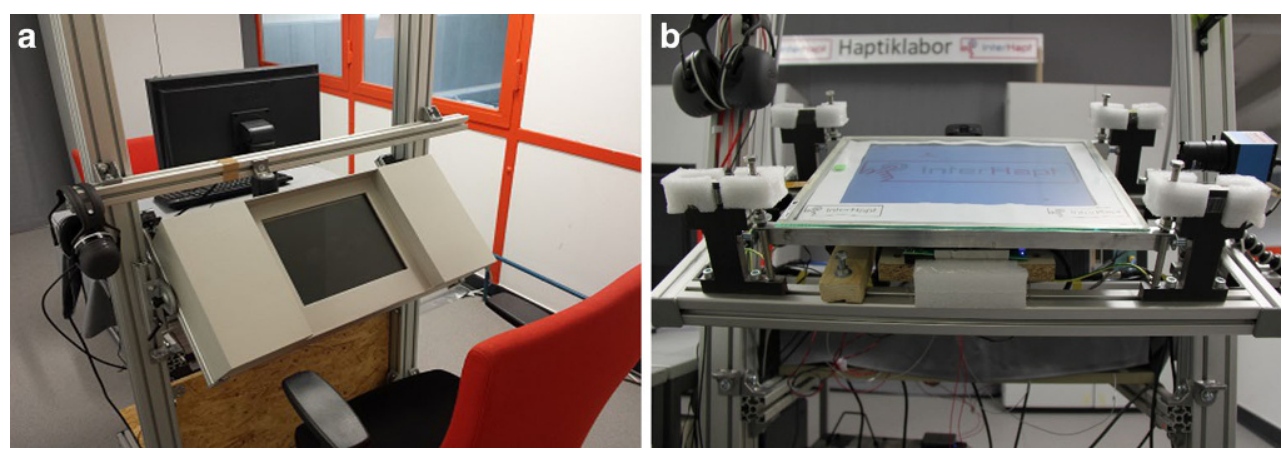
Abb. 2 Versuchs-Interface in Block B: Zusätzlich zu den beschrifteten Buttons wurde hier eine kurze Beschreibung angegeben

Fig. 2 User interface in block B: Additional descriptions were added to the labelled buttons

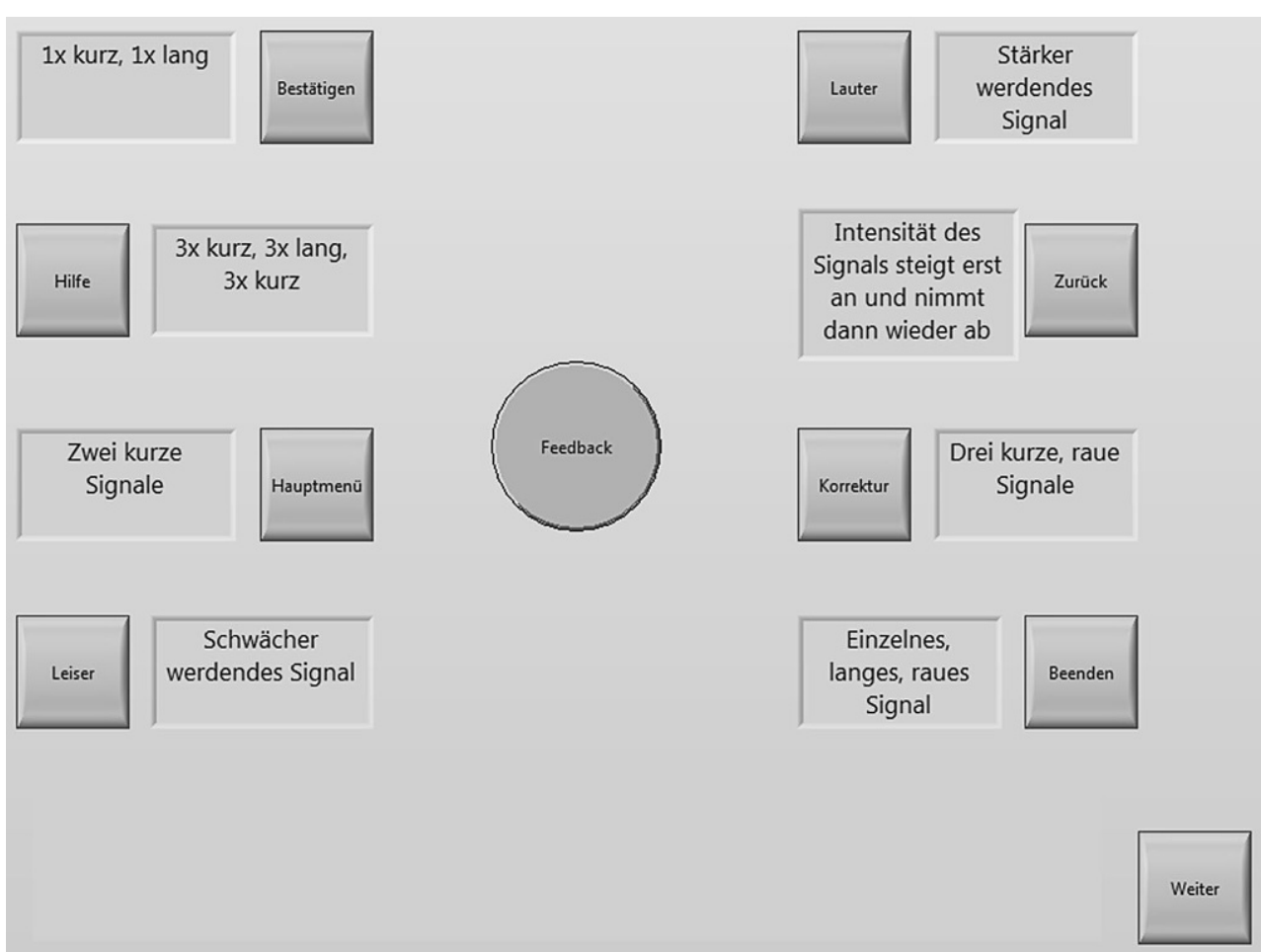

\subsection{Versuchsaufbau}

Die Evaluation des entworfenen Tactons-Sets für Standardfunktionen wurde an dem in Abb. $1 \mathrm{zu}$ sehenden Versuchsaufbau durchgeführt. Das Rahmengestell dieses Aufbaus wurde aus Item-Aluprofilen konzipiert. Auf der höhenverstellbaren Tischplatte wurde ein 15-Zoll-LC-Bildschirm angebracht. Vor diesem LC-Bildschirm wurde ein 19-Zoll-Oberflächen-kapazitives Touchpanel (3M ClearTek II mit einem USB-Controller 3M EXII-7760UC) an den nicht sensitiven Flächen allseitig verklebt. Der Metallrahmen ruht in den Lagerpunkten jeweils auf einem vorgespannten Piezo-Stapelaktor (PI Ceramic P-820.3B), welcher jeweils von einem Hochleistungs-Piezoverstärker (PI Ceramic E-617.00F) angesteuert wurde. Diese PiezoStapelaktoren wurden gewählt, um die genannten, anhand der menschlichen Wahrnehmung abgeleiteten Parameter sowie deren benötigte Bereiche darstellen zu können. Für die Messung der Frequenz und Amplitude vor und während der Untersuchung wurde ein optischer Wegsensor (KEYENCE LK-32) unterhalb des Touchpanels fest installiert, um die tatsächliche Wegänderung messen zu können.

\subsection{Versuchsdesign}

Das entstandene Tacton-Set sollte anschließend umfangreich evaluiert werden. Dafür wurde mittels LabVIEW ein Versuchsprogramm entworfen, das drei verschiedene Ver- suchsbedingungen beinhaltete, die jeder Proband zu durchlaufen hatte.

Nach einem Begrüßungsbildschirm folgte sogleich der Trainingsbildschirm. Hier hatte jeder Proband fünf Minuten Zeit, die Tactons zu den einzelnen Standardfunktionen zu erlernen. Dafür waren acht beschriftete Buttons mit einer danebenstehenden kurzen Beschreibung (wie in Abb. 2) auf dem Bildschirm verteilt, über die die einzelnen Tactons abgespielt werden konnten. Im rechten unteren Bildrand war die verbleibende Zeit zu sehen.

Nach dieser Trainingsphase wurden die Tactons unter verschiedenen Versuchsbedingungen abgefragt. Mit Aktivieren eines Buttons in der Mitte des Bildschirms wurde ein den Versuchspersonen unbekanntes Tacton abgespielt. Die Aufgabe bei der ersten Versuchsbedingung (Block A) bestand darin, dieses Tacton zu erkennen und unter den acht Antwort-Buttons die entsprechende Funktion auszuwählen, die das Tacton codiert. Jedes der acht Tactons wurde in einer randomisierten Reihenfolge dreimal abgefragt $(3 \cdot 8=24$ Einzelaufgaben in Block A), wobei sichergestellt wurde, dass ein Tacton nicht zweimal direkt hintereinander präsentiert wurde. Mit Klick auf einen Weiter-Button gelangten die Versuchspersonen zum nächsten Tacton, das korrekt erkannt werden sollte usw.

Die zweite Versuchsbedingung (Block B) war genauso aufgebaut wie die erste, mit dem einzigen Unterschied, dass hier zusätzlich zur Benennung der Funktion neben den Antwort-Buttons auch eine kurze Beschreibung der Tactons zu finden war (siehe Abb. 2). Dies sollte ausschließen, dass 
eine mögliche falsche Antwort der Probanden nur deshalb gegeben wurde, da sie sich die Tactons nicht merken konnten, und simulieren, dass die Probanden im Umgang mit den Tactons vertraut waren, z.B. da sie täglich mit ihnen in Kontakt kommen. Fehler sollten also nur gemacht werden, wenn die Tactons schlicht falsch erkannt wurden und nicht, weil deren Bedeutung verwechselt wurde. Ebenso wie in Block A wurde jedes der acht Tactons in einer randomisierten Reihenfolge dreimal abgefragt. Beide Versuchsbedingungen wurden eingangs durch ein kurzes Beispiel verdeutlicht, bevor die Versuche starteten.

Nach Durchlaufen der Blöcke A und B wurde eine kurze Pause eingelegt und der Ablauf der dritten Versuchsbedingung (Block C) erklärt. Hier wurde in einem Textfeld eine zu detektierende Funktion vorgegeben. Die Antwort-Buttons waren ohne Beschriftung oder Beschreibung auf dem Bildschirm verteilt, wobei von Aufgabe zu Aufgabe die Tactons ihre Positionen randomisiert wechselten. Das heißt, die Versuchspersonen mussten Button für Button aktivieren, bis sie das ihrer Meinung nach korrekte Tacton gefunden hatten, das die geforderte Funktion codiert. In Block $\mathrm{C}$ wurde jedes der acht Tactons zweimal in einer randomisierten Reihenfolge abgefragt, wodurch sich 16 einzeln präsentierte Tactons ergaben.

Gleichzeitig wurde den Versuchspersonen zur Ablenkung ein Video einer Fahrsituation vorgespielt, welches zudem Fragen zu dessen Inhalt enthielt, sodass der Blick der Probanden vermehrt auf den Videobildschirm gerichtet sein sollte und die Detektion des korrekten Buttons nahezu blind erfolgen musste. Die Fragen sollten von den Versuchsteilnehmern mündlich beantwortet werden, wobei die Antworten vom Versuchsleiter handschriftlich protokolliert wurden. Somit sollte das eingangs beschriebene, beispielhafte Szenario einer Bedienung des Infotainment-Systems im Auto bei gleichzeitigem Blick auf die Straße möglichst gut abgebildet werden. Die Versuchsbedingungen von Block $\mathrm{C}$ wurden ebenfalls mit einem kurzen Beispiel eingeführt, bevor die eigentlichen Versuche begonnen wurden, sodass sich die Versuchspersonen mit dieser neuen Art der Aufgabe vertraut machen konnten.

Am Anfang des Versuchs wurde vom Versuchsleiter festgelegt, welche Reihenfolge der Blöcke beim jeweiligen Probanden eingehalten wird. Dabei waren die Reihenfolgen $\mathrm{ABC}$ und $\mathrm{BAC}$ möglich. Block $\mathrm{C}$ wurde stets am Ende des Versuchs durchlaufen, da hierfür die Tactons bereits gut erlernt sein mussten.

Abschließend sollten die Tactons von den Versuchspersonen anhand von sechs Fragen subjektiv bewertet werden, wobei jeweils eine siebenstufige Likert-Skala genutzt wurde. Die Fragen lauteten im Einzelnen:

- Wie gut war die Bedeutung des Vibrationsmusters zu erlernen? (Erlernbarkeit, sehr schlecht - sehr gut)
- Wie lange haben Sie benötigt, um die Bedeutung des Vibrationsmusters zu erlernen? (Erlerndauer, sehr lange sehr kurz)

- Wie gut passt das Vibrationsmuster zur codierten Funktion? (Eignung zur Codierung, sehr schlecht - sehr gut)

- Wie empfinden Sie die Wiedererkennbarkeit des Vibrationsmusters? (Wiedererkennbarkeit, sehr schlecht - sehr gut)

- Wie angenehm empfinden Sie das Vibrationsmuster? (Annehmlichkeit, sehr unangenehm - sehr angenehm)

- Wie gut war das Vibrationsmuster von den anderen zu unterscheiden? (Unterscheidbarkeit, sehr schlecht - sehr gut)

\subsection{Versuchsdurchführung}

Nach der Begrüßung der Versuchsteilnehmer erfolgte eine Aufklärung über den Versuch und eine Einführung in das Szenario. Anschließend waren die Einverständnis- und Datenschutzerklärung sowie ein Eingangsfragebogen auszufüllen, in dem neben demographischen Daten auch die Händigkeit nach dem Edinburgh Handedness Inventory (Oldfield 1971) sowie die Technikaffinität nach Karrer et al. (2009) abgefragt wurden. Vor dem Start des eigentlichen Versuchs wurde der Versuchsstand auf die Maße der sitzenden Versuchsperson eingestellt.

Danach konnte mit der fünfminütigen Trainingsphase begonnen und, nach Klärung weiterer möglicher Fragen, der Versuch nach dem zuvor beschriebenen Vorgehen durchgeführt werden. Die vibrotaktilen Signale wurden in dieser Studie an den Zeigefinger der dominanten Hand der Versuchspersonen übermittelt. Während des Versuchs trugen die Teilnehmer sowohl In-Ear-Kopfhörer mit weißem Rauschen als auch zusätzliche Gehörkapseln, um eine auditive Beeinträchtigung durch die Geräusche der Piezoaktoren zu vermeiden. Die Durchführung nahm für einen Teilnehmer etwa 40-45 min in Anspruch.

\subsection{Ergebnisse}

54 Versuchspersonen (38 männlich, 16 weiblich) im Alter von 32,1 $\pm 14,8$ Jahren (Spannweite 18-70 Jahre) nahmen an dieser Studie teil. 48 von ihnen waren Rechtshänder, zwei Linkshänder und vier beidhändig, wobei drei den Versuch mit der rechten und einer mit der linken Hand ausführten. Bezüglich der Technikaffinität ergab sich für die Subskala „Begeisterung für Technik“ ein Wert von 3,39, für „Kompetenz im Umgang mit Technik“ ein Wert von 3,61, für „positive Technikfolgen“ ein Wert von 3,66 und für „negative Technikfolgen“ ein Wert von 3,18, wobei jeweils Werte zwischen eins (geringe Technikaffinität) bis fünf (hohe Technikaffinität) erreicht werden können (Kar- 
Abb. 3 Erkennungsraten und Standardabweichungen des Tacton-Sets (in Tabelle dargestellt als Zahl und Balkendiagramm). ERK Erkennungsrate, SD Standardabweichung

Fig. 3 Recognition rates and standard deviations of the tacton set (shown as number and bar chart). ERK recognition rate, $S D$ standard deviation

\begin{tabular}{|c|c|c|c|c|c|c|c|c|c|}
\hline & \multicolumn{2}{|c|}{$\begin{array}{c}\text { Block A } \\
\text { (nur Funktion) }\end{array}$} & \multicolumn{2}{|c|}{$\begin{array}{c}\text { Block B } \\
\text { (Funktion + } \\
\text { Beschreibung) } \\
\end{array}$} & \multicolumn{2}{|c|}{$\begin{array}{c}\text { Block C } \\
\text { (mit Ablenkung) }\end{array}$} & \multicolumn{2}{|c|}{ gesamt } \\
\hline & & ERK & SD & ERK & SD & ERK & SD & ERK & SD \\
\hline \multirow{8}{*}{ 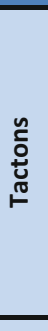 } & Beenden & 0,70 & 0,03 & 0,86 & 0,01 & 0,68 & 0,01 & 0,76 & 0,23 \\
\hline & Bestätigen & 0,57 & 0,03 & 0,65 & 0,03 & 0,53 & 0,03 & 0,59 & 0,32 \\
\hline & Hauptmenü & 0,52 & 0,05 & 0,91 & 0,03 & 0,56 & 0,03 & 0,68 & 0,24 \\
\hline & Hilfe & 0,93 & 0,03 & 0,98 & 0,02 & 0,94 & 0,01 & 0,95 & 0,13 \\
\hline & Korrektur & 0,68 & 0,05 & 0,98 & 0,02 & 0,64 & 0,01 & 0,78 & 0,23 \\
\hline & Lauter & 0,70 & 0,06 & 0,59 & 0,06 & 0,72 & 0,06 & 0,66 & 0,28 \\
\hline & Leiser & 0,65 & 0,03 & 0,69 & 0,04 & 0,60 & 0,01 & 0,65 & 0,30 \\
\hline & Zurück & 0,52 & 0,02 & 0,65 & 0,07 & 0,45 & 0,06 & 0,55 & 0,36 \\
\hline & gesamt & 0,66 & 0,24 & 0,79 & 0,16 & 0,64 & 0,24 & 0,70 & 0,19 \\
\hline
\end{tabular}

rer et al. 2009). Für die standardisierte Auswertung der Technikaffinität werden Items mit einem negativen Einfluss auf die Technikaffinität (alle Items der Subskala ,negative Technikfolgen“ sowie ein Item der Subskala „Kompetenz im Umgang mit Technik“) invertiert (Karrer et al. 2009), sodass hohe Werte einer hohen Technikaffinität entsprechen.

Die Ergebnisse von 3456 einzelnen Messungen ([24 aus Block A+24 aus Block B +16 aus Block C] 54 Versuchspersonen) wurden in die Auswertung einbezogen, wobei eine Gesamterkennungsrate von $0,70 \pm 0,19$ erreicht wurde. Einer Versuchsperson gelang es, alle Tactons korrekt zu erkennen und zwei Versuchspersonen machten während ihrer 64 Einzelmessungen nur einen Fehler. Die schlechteste Erkennungsrate lag bei 0,34 und war das Ergebnis zweier Versuchsteilnehmer. Abb. 3 sind die einzelnen Erkennungsraten der acht Tactons unter den jeweiligen Versuchsbedingungen zu entnehmen (zur schnelleren Erfassung der Unterschiede sind diese auch grafisch in Form von Balken dargestellt). Es fällt auf, dass außer bei der Funktion Lauter die Erkennungsraten eines jeden Tactons in Block B am besten waren. Insgesamt wurde die Funktion Hilfe am besten und Zurück am schlechtesten erkannt.

Zur Überprüfung statistisch relevanter Unterschiede werden nichtparametrische Tests herangezogen, da bei den zu untersuchenden Erkennungsraten laut Shapiro-Wilk-Test nicht durchgängig eine Normalverteilung angenommen werden kann.

Bezüglich des Geschlechts ist kein signifikanter Einfluss auf die Erkennungsraten festzustellen - weder insgesamt, noch in den einzelnen Blöcken A, B und C, wie ein MannWhitney-U-Test zeigte. Vergleicht man allerdings die Erkennungsraten von jüngeren und älteren Versuchsteilnehmern (Vergleich des unteren und oberen Quartils) mit ebendiesem Test, zeigen sich hier insgesamt $(p=0,001)$ und in den einzelnen Blöcken (A: $p=0,004, \mathrm{~B}: p=0,002, \mathrm{C}$ : $p=0,001)$ signifikante Unterschiede, wobei stets die jüngeren Versuchsteilnehmer bessere Ergebnisse erzielten als die älteren.
Die Reihenfolge, in der die einzelnen Blöcke präsentiert wurden (ABC oder BAC), hat laut durchgeführtem MannWhitney-U-Test keinen signifikanten Einfluss auf die Erkennungsraten.

Es gibt aber statistisch bedeutsame Unterschiede in den Erkennungsraten der einzelnen Blöcke. Ein Friedman-Test und Post-Hoc-Tests mit Bonferroni-Korrektur zeigen signifikante Unterschiede zwischen Block A und Block B $(p<0,001)$ sowie zwischen Block B und Block C $(p<0,001)$, wobei in beiden Fällen in Block B die besseren Ergebnisse erzielt werden. Zwischen Block A und Block C konnte kein statistisch bedeutsamer Unterschied festgestellt werden.

\subsection{Verbesserungspotenzial}

Die Ergebnisse zeigen, dass sich die Erkennungsraten der einzelnen Tactons teils stark unterscheiden, was auf ein gewisses Verbesserungspotenzial für die Gestaltung einzelner Tactons schließen lässt.

In vergleichbaren Studien werden Erkennungsraten von $80 \%$ als geeignet und von über $90 \%$ als ",nahezu perfekt" angesehen (Brown et al. 2005, 2006). Legt man Block B zugrunde, in dem durch die Beschreibung der Tactons den Versuchspersonen eine derartige Hilfestellung gegeben wurde, als wären sie mit den Tactons durch alltäglichen Umgang bereits vertraut, so wären vier der acht Tactons (Beenden, Hauptmenü, Hilfe und Korrektur) geeignet drei davon sogar mit Erkennungsraten von über $90 \%$. Bei den übrigen vier Tactons (Bestätigen, Lauter, Leiser und Zurück) sollte die Gestaltung nochmals überdacht und gegebenenfalls angepasst werden.

Interessant ist die Beobachtung, dass die Erkennungsraten von Block $\mathrm{A}$ und Block $\mathrm{C}$ bei jeder der acht Funktionen vergleichbar sind - es sind keine signifikanten Unterschiede festzustellen. Es wäre zu erwarten gewesen, dass die Erkennungsraten in Block $\mathrm{C}$ aufgrund der zusätzlichen Sekundäraufgabe schlechter ausfallen als in Block A. Mög- 
Tab. 1 Verwechslungsmatrix des Tacton-Sets

Table 1 Confusion matrix of the tacton set

\begin{tabular}{|c|c|c|c|c|c|c|c|c|}
\hline \multirow{2}{*}{$\begin{array}{l}\text { Genanntes } \\
\text { Tacton }\end{array}$} & \multicolumn{8}{|c|}{ Korrektes Tacton } \\
\hline & Beenden & Bestätigen & Hauptmenü & Hilfe & Korrektur & Lauter & Leiser & Zurück \\
\hline Beenden & 0,76 & 0,04 & 0,02 & 0,00 & 0,02 & 0,11 & 0,16 & 0,04 \\
\hline Bestätigen & 0,02 & 0,59 & 0,14 & 0,00 & 0,05 & 0,02 & 0,02 & 0,10 \\
\hline Hauptmenї & 0,03 & 0,20 & 0,68 & 0,01 & 0,05 & 0,02 & 0,03 & 0,09 \\
\hline Hilfe & 0,01 & 0,00 & 0,01 & 0,95 & 0,02 & 0,00 & 0,00 & 0,00 \\
\hline Korrektur & 0,04 & 0,03 & 0,04 & 0,03 & 0,78 & 0,09 & 0,03 & 0,05 \\
\hline Lauter & 0,05 & 0,02 & 0,02 & 0,01 & 0,03 & 0,66 & 0,06 & 0,05 \\
\hline Leiser & 0,07 & 0,03 & 0,03 & 0,00 & 0,02 & 0,03 & 0,65 & 0,12 \\
\hline Zurück & 0,03 & 0,08 & 0,06 & 0,01 & 0,04 & 0,05 & 0,06 & 0,55 \\
\hline
\end{tabular}

licherweise wird der Nachteil der Ablenkung durch den einsetzenden Lerneffekt aufgehoben, da Block C stets am Ende des Versuchs nach dem Absolvieren von Block A und Block B (oder andersherum) durchlaufen wurde.

Dieser erste Entwurf eines vollständigen Tacton-Sets für Standardfunktionen scheint aber bereits eine geeignete Ausgangsbasis für eine anschließende, schrittweise Verbesserung zu sein. Die guten Erkennungsraten einiger Tactons gerade in Block B - lassen darauf schließen, dass das Tacton-Set bei der Interaktion mit einem Touchscreen durchaus einen Mehrwert liefern kann.

Nach genauer Analyse der Erkennungsraten und Verwechslungen mit anderen Tactons, dargestellt in Tab. 1, sowie einer anschließenden Überarbeitung des Tacton-Sets soll dieser Versuch nun wiederholt werden, um die Erkennungsraten der einzelnen - und besonders der in diesem Versuch weniger gut erkannten - Tactons zu verbessern.

\section{Verbesserung des Tacton-Sets für Standardfunktionen}

Das nach der vorherigen Evaluation identifizierte Verbesserungspotenzial bezüglich der Erkennbarkeit einzelner Tactons und der Unterscheidbarkeit untereinander soll nun umgesetzt werden. Daher wird im Folgenden die Ausgestaltung der vier weniger gut erkannten Tactons Bestätigen, Lauter, Leiser und Zurück analysiert und entsprechend der Erkenntnisse aus dem vorherigen Versuch angepasst. Anschließend wird das gesamte Tacton-Set erneut evaluiert, wobei das gleiche Versuchsdesign wie im vorherigen Versuch gewählt wird, um die Vergleichbarkeit der Ergebnisse zu gewährleisten. Durch ein solches Vorgehen kann das Tacton-Set für Standardfunktionen Schritt für Schritt verbessert und die Ergebnisse jeder Iteration können miteinander verglichen werden.

Die nachfolgend beschriebene Studie beinhaltet also die erste von weiteren möglichen Iterationen einer schrittwei- sen Optimierung dieses Tacton-Sets für Standardfunktionen.

\subsection{Weiterentwicklung des Tacton-Sets für Standardfunktionen}

Sollte das Tacton Bestätigen erkannt werden, und wurde seitens der Versuchsteilnehmer eine falsche Antwort gegeben, so lautete diese meist Hauptmenü (50\%). Beide Funktionen wurden durch zwei Vibrationen mit einer Frequenz von je $150 \mathrm{~Hz}$ sowie einer Pause dazwischen codiert. Die unterschiedliche Dauer der beiden Tactons (Bestätigen: $580 \mathrm{~ms}$, Hauptmenü: $400 \mathrm{~ms}$ ) sowie der unterschiedliche Rhythmus (Bestätigen: kurz-lang, Hauptmenü: kurzkurz) scheinen für eine eindeutige Differenzierung nicht auszureichen. Daher wird das Tacton Bestätigen nun durch eine kurze, $100 \mathrm{~ms}$ andauernde Vibration mit einer Frequenz von $100 \mathrm{~Hz}$ codiert. Somit ist einerseits eine bessere Unterscheidbarkeit gegenüber der Funktion Hauptmenü gegeben, andererseits konnten die Vorschläge aus der Gruppenbefragung noch besser umgesetzt werden, da für die Funktion Bestätigen z.B. ein „einfaches Klicken“ bzw. ein „kurzes, einfaches Klopfen/Klacken/Klingen“ vorgeschlagen wurden. Auch die positive Konnotation dieser Funktion wird mit der als angenehm wahrgenommenen Frequenz von $100 \mathrm{~Hz}$ beibehalten (Stein et al. 2016), wobei im Vergleich der Frequenzen der Tactons Bestätigen und Hauptmenü $(150 \mathrm{~Hz})$ die für eine gute Unterscheidbarkeit geforderte Unterschiedsschwelle von 1,5 (Stein et al. 2017) eingehalten wird.

Der Vergleich des Tactons Lauter mit dem Tacton Korrektur, welches die häufigste falsche Antwort im vorherigen Versuch war (35\%), zeigt, dass beide Tactons aus drei einzelnen Vibrationen mit einer Frequenz von $250 \mathrm{~Hz}$ konzipiert waren. Während die Intensität der drei Vibrationen des Tactons Lauter anstieg, wies Korrektur eine gleichbleibende Intensität auf. Zudem wurde das Tacton Korrektur durch eine zusätzliche Modulationsfrequenz von $30 \mathrm{~Hz}$ rauer gestaltet. Sowohl die unterschiedlichen Intensitäten als auch die 
Differenzierung bezüglich der Rauheit scheinen für die Versuchspersonen nicht eindeutig unterscheidbar gewesen $\mathrm{zu}$ sein. Auch die Unterschiede in der Dauer (Lauter: $450 \mathrm{~ms}$, Korrektur: $700 \mathrm{~ms}$ ) waren offenbar nicht ausreichend. Bei der Weiterentwicklung des Tactons Lauter muss aber auch das Tacton Leiser in die Überlegungen einbezogen werden, da diese Tactons als gegensätzliches Paar entworfen werden sollten.

Das Tacton Leiser wurde überwiegend mit dem Tacton Beenden verwechselt (48\%). Beenden wurde durch eine einzelne, $500 \mathrm{~ms}$ lange Vibration von $250 \mathrm{~Hz}$, moduliert mit $50 \mathrm{~Hz}$, codiert. Die durchgehende, raue Vibration des Tactons Beenden konnte demnach nicht gut von den schwächer werdenden Vibrationen des Tactons Leiser differenziert werden. Daher wurde das Tacton Leiser folgendermaBen neu codiert. Auf eine Vibration von $250 \mathrm{~Hz}$ mit $80 \mathrm{~ms}$ Dauer folgte eine $200 \mathrm{~Hz}$ Vibration von einer Dauer von $120 \mathrm{~ms}$ sowie eine Vibration von $150 \mathrm{~Hz}$ mit $160 \mathrm{~ms}$ Dauer. Diese Vibrationen nahmen nach wie vor in ihrer Intensität ab. Zwischen den drei Vibrationen wurde nun allerdings noch eine Pause von jeweils $20 \mathrm{~ms}$ eingefügt, um die unterschiedlichen Frequenzen, Intensitäten und Dauern der einzelnen Vibrationen dieses Tactons trennschärfer wahrnehmbar zu machen. Die Gesamtdauer des Tactons Leiser konnte im Vergleich zum ersten Entwurf dieses Tactons um $50 \mathrm{~ms}$ reduziert werden, wobei die Vorschläge aus der Gruppenbefragung sogar in höherem Maße umgesetzt wurden, da dieses Tacton nun sowohl durch eine abnehmende Intensität als auch eine abnehmende Frequenz codiert wurde.

Das Tacton Lauter wurde, wie im vorherigen Versuch, analog zum Tacton Leiser in umgekehrter Reihenfolge gestaltet, beginnend mit der schwächsten und kürzesten Vibration bis hin zur stärksten und längsten Vibration.

Das Tacton Zurück wurde am häufigsten mit Leiser (35\%) und zudem zu $11 \%$ mit Lauter verwechselt. Während Lauter und Leiser aus jeweils drei Vibrationsmustern, die in ihrer Intensität anstiegen bzw. abnahmen, bei einer Frequenz von $250 \mathrm{~Hz}$ gestaltet wurden, wurde die Funktion Zurück bei ebendieser Frequenz mit einer zusätzlichen Modulationsfrequenz von $50 \mathrm{~Hz}$ codiert. Zurück bestand hierbei aus vier in ihrer Intensität ansteigenden Vibrationen und vier schwächer werdenden Vibrationen mit einer Pause dazwischen. Nach der bereits beschriebenen Neugestaltung der Tactons Lauter und Leiser unterscheiden sich diese bereits stärker vom Tacton Zurück als zuvor. Die Dauer der einzelnen Vibrationen variiert nun, zudem sind die einzelnen auf- und absteigenden Vibrationen bei Lauter und Leiser durch Pausen getrennt, was bei dem Tacton Zurück nicht der Fall ist. Daher wird der Tacton Zurück an dieser Stelle nur noch durch Weglassen einer aufsteigenden und einer absteigenden Vibration vereinfacht und dadurch in seiner Dauer um $250 \mathrm{~ms}$ auf eine Gesamtdauer von $650 \mathrm{~ms}$ verkürzt. Auch die in der Gruppenbefragung zusammen- getragenen Vorschläge, die häufig ein ,Vorwärts“ und ein anschließendes „Rückwärts“ beinhalten, sowie die geäuBerte negative Konnotation dieser Funktion, welche durch die Rauheit $(250 \mathrm{~Hz}$ moduliert mit $50 \mathrm{~Hz}$, siehe (Brown et al. 2005)) berücksichtigt wurde, werden beibehalten.

Zusammenfassend wurden die Ergebnisse des vorherigen Versuchs genutzt, um das Tacton-Set dahingehend weiterzuentwickeln, dass die Unterschiede zwischen den einzelnen Tactons größer sind, um somit bessere Erkennungsraten aufgrund weniger Verwechslungen zu erzielen. Im Einzelnen wurden die vier zuvor am seltensten richtig identifizierten Tactons ausgewählt, ihre Verwechslungen mit anderen Tactons analysiert und die Gestaltung dieser Tactons neu umgesetzt, wobei nach wie vor existierende Erkenntnisse (z.B. Brewster und Brown 2004; Brown et al. 2006; Constantin und Brewster 2010; Stein et al. 2017; van Erp 2002) berücksichtigt wurden.

\subsection{Versuchsdesign und -durchführung}

Sowohl das Versuchsdesign als auch die Versuchsdurchführung sind identisch zum zuvor vorgestellten Versuch, um die Vergleichbarkeit der Ergebnisse und der Erkennungsraten des ursprünglichen und des weiterentwickelten TactonSets zu gewährleisten. Um einen möglichen Trainingseffekt als Störvariable zu vermeiden, wird zur Durchführung dieses zweiten Versuchs bewusst eine Stichprobe gebildet, die keine Person enthält, welche am ersten, bereits vorgestellten Versuch teilgenommen hat.

\subsection{Ergebnisse}

An dieser Studie nahmen 39 Versuchspersonen (25 männlich, 14 weiblich) im Alter von 31,1 $\pm 13,5$ Jahren (Spannweite 18-82 Jahre) teil. 34 von ihnen waren Rechtshänder, vier Linkshänder und ein Teilnehmer war beidhändig, wobei er den Versuch mit der rechten Hand ausführte. Die Technikaffinität (Karrer et al. 2009) ergab für die Subskala „Begeisterung für Technik“ einen Wert von 3,14, für „,Kompetenz im Umgang mit Technik“ einen Wert von 3,65, für „positive Technikfolgen“ ebenfalls einen Wert von 3,65 und für ,negative Technikfolgen“ einen Wert von 3,13.

Die Gesamterkennungsrate dieser Studie lag bei $0,79 \pm$ 0,18, wobei 2496 Einzelmessungen ([24 aus Block A+24 aus Block B +16 aus Block C]·39 Versuchspersonen) in die Auswertung einbezogen wurden. Drei Versuchspersonen konnten alle Tactons korrekt erkennen und zwei Versuchspersonen machten in ihren 64 einzelnen Messungen lediglich einen Fehler. Die schlechteste Erkennungsrate eines Probanden lag bei 0,38. Die Erkennungsraten der einzelnen Tactons unter den verschiedenen Versuchsbedingungen sind Abb. 4 zu entnehmen (als Zahl und Balkengrafik dargestellt). Es zeigt sich, dass die Erkennungsraten jedes 
Abb. 4 Erkennungsraten und Standardfunktionen des weiterentwickelten Tactons-Sets (in Tabelle dargestellt als Zahl und Balkendiagramm). ERK Erkennungsrate, $S D$ Standardabweichung

Fig. 4 Recognition rates and standard deviations of the advanced tacton set (shown as number and bar chart). $E R K$ recognition rate, $S D$ standard deviation

\begin{tabular}{|c|c|c|c|c|c|c|c|c|c|}
\hline & \multicolumn{2}{|c|}{$\begin{array}{c}\text { Block A } \\
\text { (nur Funktion) }\end{array}$} & \multicolumn{2}{|c|}{$\begin{array}{c}\text { Block B } \\
\text { (Funktion + } \\
\text { Beschreibung) }\end{array}$} & \multicolumn{2}{|c|}{$\begin{array}{c}\text { Block C } \\
\text { (mit Ablenkung) }\end{array}$} & \multicolumn{2}{|c|}{ gesamt } \\
\hline & & ERK & SD & ERK & SD & ERK & SD & ERK & SD \\
\hline \multirow{8}{*}{ 气ั: } & Beenden & 0,71 & 0,11 & 0,86 & 0,07 & 0,64 & 0,13 & 0,75 & 0,25 \\
\hline & Bestätigen & 0,92 & 0,06 & 0,91 & 0,06 & 0,79 & 0,08 & 0,88 & 0,20 \\
\hline & Hauptmenü & 0,79 & 0,06 & 0,84 & 0,09 & 0,79 & 0,10 & 0,81 & 0,22 \\
\hline & Hilfe & 0,97 & 0,04 & 0,99 & 0,01 & 0,95 & 0,05 & 0,97 & 0,07 \\
\hline & Korrektur & 0,84 & 0,09 & 0,80 & 0,06 & 0,62 & 0,00 & 0,77 & 0,25 \\
\hline & Lauter & 0,89 & 0,06 & 0,86 & 0,05 & 0,79 & 0,05 & 0,86 & 0,20 \\
\hline & Leiser & 0,64 & 0,08 & 0,68 & 0,09 & 0,73 & 0,06 & 0,68 & 0,30 \\
\hline & Zurück & 0,63 & 0,04 & 0,60 & 0,02 & 0,49 & 0,03 & 0,58 & 0,34 \\
\hline & gesamt & 0,80 & 0,20 & 0,82 & 0,19 & 0,73 & 0,24 & 0,79 & 0,18 \\
\hline
\end{tabular}

Tactons in Block C am schlechtesten waren. Insgesamt wurde die Funktion Hilfe am besten und die Funktion Zurück am schlechtesten erkannt.

Laut einem durchgeführter Shapiro-Wilk-Test sind die zu untersuchenden Erkennungsraten nicht normalverteilt, daher wird im Folgenden erneut auf nichtparametrische Tests zurückgegriffen, um statistisch signifikante Unterschiede zu identifizieren.

Der Vergleich der Erkennungsraten männlicher und weiblicher Versuchsteilnehmer mittels Mann-WhitneyU-Test lieferte sowohl für die Gesamterkennungsrate $(p=0,005)$ als auch für die Blöcke B $(p<0,001)$ und C $(p=0,009)$ statistisch bedeutsame Unterschiede. Männliche Versuchsteilnehmer erzielten hier jeweils die besseren Ergebnisse. In Block A konnte keine Signifikanz nachgewiesen werden. Bezüglich des Alters sind mit dem gleichen Test signifikante Effekte in den Erkennungsraten jüngerer und älterer Versuchspersonen sowohl bei der Gesamterkennungsrate $(p=0,024)$ als auch in den Blöcken A $(p=0,020)$ und $\mathrm{C}(p=0,013)$ festzustellen, wobei die jüngeren Teilnehmer jeweils bessere Ergebnisse erreichten als die älteren. In Block B konnten keine signifikanten Unterschiede im Altersvergleich festgestellt werden.

Die Reihenfolge der Präsentation der einzelnen Blöcke (ABC oder BAC) hat laut durchgeführten Mann-WhitneyU-Test erneut keinen statistisch bedeutsamen Einfluss auf die Erkennungsraten.

Im Vergleich der Erkennungsraten der einzelnen Blöcke sind deutliche Unterschiede festzustellen. Ein Friedman-Test lässt die Nullhypothese zwar ablehnen, aber anschließende Post-Hoc-Tests mit Bonferroni-Korrektur liefern keine signifikanten paarweisen Unterschiede zwischen den einzelnen Blöcken.

\subsection{Vergleich der beiden Studien}

Aufgrund des identischen Versuchsdesigns (geändert wurde lediglich die Gestaltung von vier der acht Tactons) und einer vergleichbaren Probandenpopulation hinsichtlich Alter,
Geschlecht und Technikaffinität sind die Ergebnisse des zuletzt vorgestellten Versuchs mit denen des vorherigen vergleichbar. Da außerdem keine Versuchsperson an beiden Evaluationen teilnahm, kann ein Lerneffekt als mögliche Störvariable ausgeschlossen werden.

Beim Vergleich der Erkennungsraten der beiden Versuche fällt auf, dass sich die Gesamterkennungsrate von $0,70 \pm 0,19$ im ersten auf $0,79 \pm 0,18$ im zweiten Versuch verbessert hat. Auch in jedem einzelnen der drei Blöcke wurden insgesamt bessere Erkennungsraten erzielt. Dieser Unterschied war in Block A am größten, die Erkennungsrate steigerte sich hier von $0,66 \pm 0,24$ auf $0,80 \pm 0,20$ (Block B: von $0,79 \pm 0,16$ auf $0,82 \pm 0,19$; Block $\mathrm{C}$ von $0,64 \pm 0,24$ auf $0,73 \pm 0,24)$.

Diese Beobachtungen werden durch die Untersuchung statistisch relevanter Effekte untermauert. Ein Mann-Whitney-U-Test liefert sowohl für die Gesamterkennungsrate $(p=0,038)$ als auch für den Block A $(p=0,004)$ signifikante Unterschiede, wobei das weiterentwickelte Tacton-Set jeweils die besseren Ergebnisse erzielte. Für die Blöcke B und $\mathrm{C}$ konnten hingegen keine signifikanten Unterschiede festgestellt werden.

Bezüglich der Erkennungsraten der einzelnen Tactons konnten ebenfalls interessante Effekte aufgezeigt werden. So zeigte ein durchgeführter Mann-Whitney-U-Test, dass bei den Tactons Bestätigen $(p<0,001)$, Hauptmenü $(p=0,005)$ und Lauter $(p=0,001)$ signifikante Unterschiede im Vergleich der Erkennungsraten über alle drei Blöcke $\mathrm{zu}$ beobachten sind, wobei auch hier das weiterentwickelte Tacton-Set stets die höheren Erkennungsraten aufweist. Bei den anderen fünf Tactons sind keine statistisch bedeutsamen Unterschiede festzustellen.

Bei den subjektiven Beurteilungen der acht Tactons durch die Versuchspersonen hat sich das Tacton Bestätigen nach der Weiterentwicklung in allen abgefragten Dimensionen signifikant verbessert (jeweils $p<0,001$; siehe auch Abb. 5) wie ein durchgeführter Mann-Whitney-UTest zeigt. Auch das Tacton Hauptmenü zeigte signifikant bessere Beurteilungen bei der Erlernbarkeit $(p=0,040)$, 
Abb. 5 Exemplarischer Vergleich der subjektiven Beurteilung des Tactons Bestätigen Fig. 5 Exemplary comparison of the subjective evaluation of the tacton $O K /$ confirm

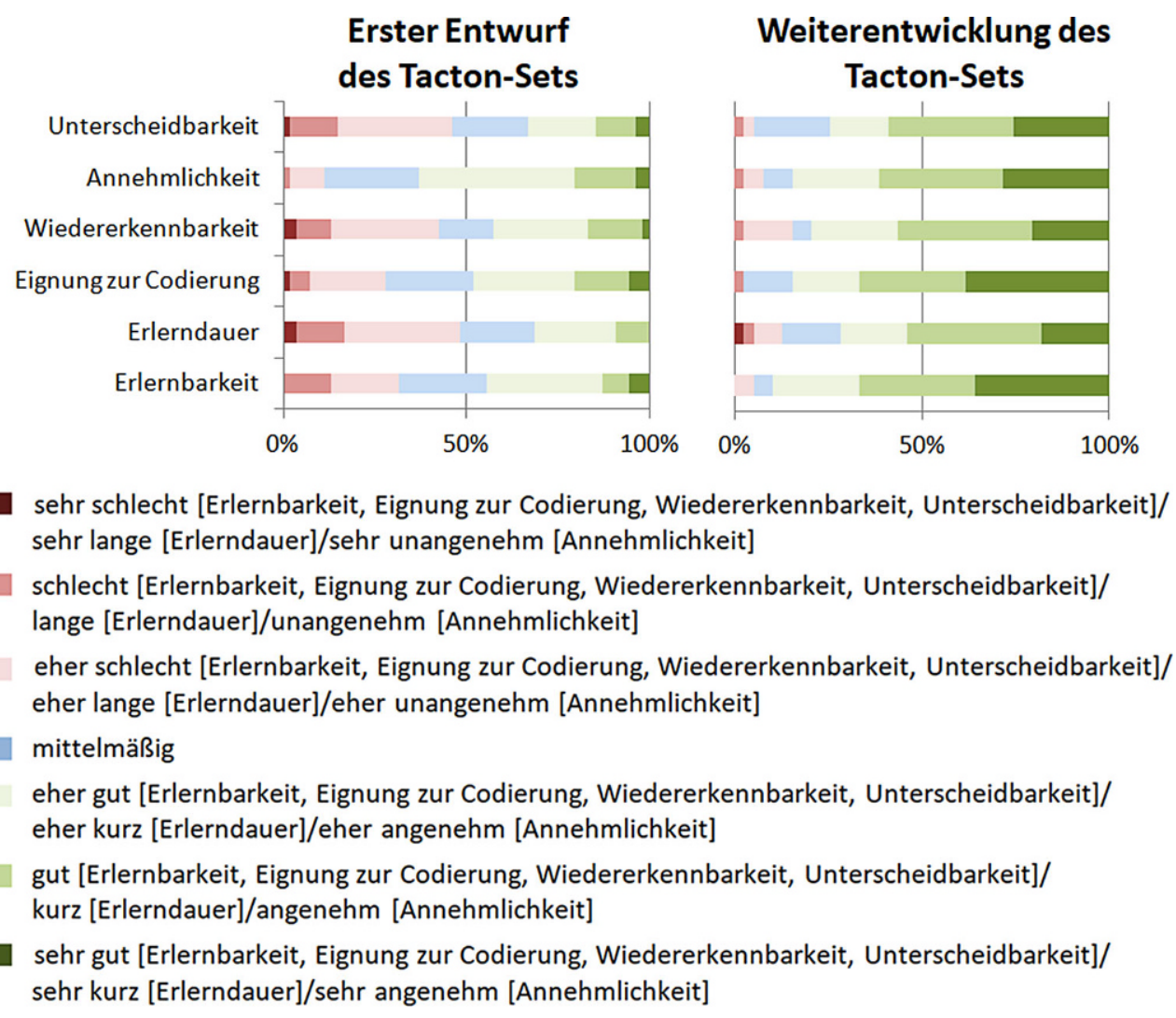

der Annehmlichkeit $(p=0,041)$ und der Unterscheidbarkeit $(p=0,002)$. Das Tacton Zurück hingegen wurde nach der Weiterentwicklung von den Versuchspersonen bezüglich Erlernbarkeit $(p=0,033)$, Eignung zur Codierung $(p=0,020)$, Wiedererkennbarkeit $(p=0,020)$ und Unterscheidbarkeit $(p=0,027)$ schlechter beurteilt. Für die weiteren Tactons konnten keine signifikanten Effekte in einer der abgefragten Dimensionen festgestellt werden.

\section{Diskussion}

Das in Abschn. 3 entworfene Tacton-Set für Standardfunktionen konnte durch die beschriebene Weiterentwicklung (siehe Abschn. 4) deutlich verbessert werden. Die Gesamterkennungsrate wurde signifikant um $9 \%$ gesteigert (von $0,70 \pm 0,19$ auf $0,79 \pm 0,18$ ), und auch die Erkennungsraten der einzelnen Blöcke wurden erhöht.

Vergleichbare Erkennungsraten wurden in früheren Studien erzielt. Azadi und Jones (2014) entwarfen beispielsweise neun Tactons aus den Parametern Frequenz, Amplitude und Dauer und erzielten zunächst Erkennungsraten am Zeigefinger von $73 \%$. Nach einer Anpassung der neun Tactons, bei denen nun die Parameter Frequenz, Amplitude und Rhythmus genutzt wurden, stieg die Erkennungsrate auf $79 \%$ (Azadi und Jones 2014). Pescara et al. (2019) erreichten in ihrer Studie, in der durch ein vibrotaktiles Arm- band Veränderungen volatiler Märkte (z.B. von Aktien) als Ergänzung einer Smartphone-Anwendung genutzt wurden, Erkennungsraten von 84\%. Auch Luzhnica et al. (2016) erzielten zunächst eine Gesamterkennungsrate von $81 \%$, wenn die Vibrationsmuster an der Hand appliziert wurden. Nach einer mehrstündigen Trainingsphase stieg diese Erkennungsrate auf $85 \%$ bzw. 96\% - je nach Komplexität der Signale (Luzhnica et al. 2016).

Bei der vorliegenden Untersuchung fällt auf, dass sich die Erkennungsraten unter den verschiedenen Versuchsbedingungen, insbesondere zwischen den Blöcken A und B, nun weniger unterscheiden. Während bei der Evaluation des ersten Tacton-Sets die in Block B erzielten Ergebnisse signifikant besser waren als die der Blöcke $\mathrm{A}$ und $\mathrm{C}$, so sind nach der Evaluation des weiterentwickelten TactonSets keine signifikanten Unterschiede in den Erkennungsraten der einzelnen Blöcke mehr nachzuweisen. Bemerkenswert ist diese Erkenntnis vor allem für Block C (Ablenkung), was dafür spricht, dass bei entsprechender Ausgestaltung der Tactons unterschiedliche Aufgaben über die verschiedenen Sinneskanäle verteilt werden können (Freeman et al. 2017), und frühere Erkenntnisse bestätigt. Auch in der Studie von Luzhnica und Veas (2019) waren die Ergebnisse unter Ablenkung zwar etwas schlechter, die Erkennungsraten aber nicht signifikant geringer als ohne eine ablenkende Nebenaufgabe. In der vorliegenden Studie könnte dies auf eine allgemein bessere Unterscheidbarkeit 
der Tactons zurückzuführen sein, da zum einen in Block A (absolutes Erkennen der Tactons ist gefragt, ohne Hilfestellung oder Ablenkung) die größten Steigerungen in den Erkennungsraten erreicht werden konnten und die Hilfestellung in Block B kaum noch zum Tragen kommt. Stattdessen ist nun der Einfluss der Ablenkung in Block C deutlicher zu erkennen. Zum anderen wurden insbesondere bei den zuvor schwierig zu unterscheidenden Tactons, wie z.B. Bestätigen und Hauptmenü, über alle Versuchsbedingungen hinweg große Verbesserungen in den Erkennungsraten erzielt (Gesamterkennungsraten: Bestätigen von $0,59 \pm 0,32$ auf $0,88 \pm 0,20$, Hauptmenü von $0,68 \pm 0,24$ auf $0,81 \pm 0,22)$. Zudem wurden diese beiden Tactons nach der Weiterentwicklung des Tacton-Sets auch subjektiv besser bewertet. Bemerkenswert ist an dieser Stelle außerdem, dass das Tacton Hauptmenü auch ohne Modifikation eine deutlich bessere Erkennungsrate erzielen konnte, was dafür spricht, dass das Prüfen der Verwechslungsraten durchaus sinnvoll ist, um Verbesserungspotenziale zu identifizieren, und die vorgenommenen Änderungen Wirkung zeigten.

Bei den ebenfalls häufig untereinander verwechselten Tactons Lauter, Leiser und Zurück konnte Lauter eine deutliche Steigerung der Erkennungsrate erzielen (von $0,66 \pm 0,28$ auf $0,86 \pm 0,20$ ). Leiser und Zurück verbesserten sich hingegen nur minimal um jeweils $3 \%$. In Verbindung mit der Tatsache, dass diese beiden Tactons auch in der absoluten Erkennungsrate am schlechtesten abschnitten, kann hier von weiterem Verbesserungspotenzial bezüglich deren Ausgestaltung ausgegangen werden, was in einer weiteren Iteration umgesetzt werden könnte. Zudem wurde das Tacton Zurück nach der Weiterentwicklung von den Versuchspersonen als signifikant schlechter beurteilt, obwohl die Erkennungsraten vergleichbar waren. Warum sich die Erkennungsraten des Tactons Lauter derart steigern konnten, wohingegen die des Tactons Leiser keine Verbesserungen zeigte, kann nicht abschließend geklärt werden. Möglicherweise liefert die gegensätzliche Gestaltung dieser beiden Tactons hierfür eine Erklärung. Die lange, schwache Vibration am Anfang des Tactons Lauter kann gut wahrgenommen werden und die Steigerung hin zur kurzen, starken Vibration am Ende des Tactons ist deutlich spürbar. Demgegenüber könnte die starke Vibration zu Beginn des Tactons Leiser die Wahrnehmung der folgenden, schwächeren Vibrationen erschweren und somit zu Verwechslungen mit den ebenfalls anhand einer Modulation der Intensität gestalteten Tactons Lauter und Zurück führen. Dieser Umstand würde den großen Unterschied der Erkennungsraten von Lauter gegenüber Leiser und Zurück erklären.

Alle Verwechslungsraten des weiterentwickelten TactonSets sind in der folgenden Tab. 2 dargestellt.

Bezüglich der grundlegenden Eignung des weiterentwickelten Tacton-Sets konnten deutliche Verbesserungen erzielt werden. Zieht man erneut Block B heran, in dem durch eine Hilfestellung (zusätzliche Beschreibung der Tactons neben den Antwort-Buttons) simuliert wurde, dass die Ausgestaltung der Tactons den Versuchspersonen bekannt ist, so erreichten sechs der acht Tactons (Beenden, Bestätigen, Hauptmenü, Hilfe, Korrektur und Lauter) Erkennungsraten von über $80 \%$, was als geeignet angesehen werden kann (Brown et al. 2005, 2006).

Zum Versuchsdesign ist einschränkend zu sagen, dass aufgrund der unterschiedlichen Aufgaben der Blöcke A und B im Vergleich zu Block C die Erkennungsraten nur eingeschränkt miteinander zu vergleichen sind. Um auch unter Ablenkung eine Aussage treffen zu können, ob die Tactons unter Simulation des alltäglichen Umgangs durch die Hilfestellung (wie in Abb. 2) besser erkannt werden, könnte ein Block D dem Versuchsdesign hinzugefügt werden. In diesem Block D wäre die Aufgabe gleich der in Block C, allerdings wäre hier eine ebensolche Hilfestellung wie in Block B gegeben. Ein zusätzlicher Versuchsblock hätte aber den zeitlichen Rahmen für die Versuchsdurchführung gesprengt.

Es ist außerdem zu erwähnen, dass den Versuchsteilnehmern lediglich eine fünfminütige Übungsphase zur Verfügung stand, um die Tactons und deren zugeordnete Funktionen zu erlernen. Wäre diese Übungsphase länger oder - eine erfolgte Integration der Tactons in unterschiedliche Geräte vorausgesetzt - würde ein täglicher Umgang mit diesen Tactons stattfinden, so würden die Erkennungsraten

Tab. 2 Verwechslungsmatrix des weiterentwickelten Tacton-Sets

Table 2 Confusion matrix of the advanced tacton set

\begin{tabular}{lllllllll}
\hline \multirow{2}{*}{$\begin{array}{l}\text { Genanntes } \\
\text { Tacton }\end{array}$} & \multicolumn{1}{l}{ Korrektes Tacton } & & & & & \\
\cline { 2 - 8 } & Beenden & Bestätigen & Hauptmenü & Hilfe & Korrektur & Lauter & Leiser & Zurück \\
\hline Beenden & $\mathbf{0 , 7 5}$ & 0,04 & 0,03 & 0,00 & 0,04 & 0,02 & 0,08 & 0,04 \\
Bestätigen & 0,05 & $\mathbf{0 , 8 8}$ & 0,02 & 0,00 & 0,01 & 0,01 & 0,06 & 0,02 \\
Hauptmenü & 0,03 & 0,02 & $\mathbf{0 , 8 1}$ & 0,00 & 0,06 & 0,02 & 0,02 & 0,10 \\
Hilfe & 0,00 & 0,00 & 0,01 & $\mathbf{0 , 9 7}$ & 0,02 & 0,00 & 0,00 & 0,03 \\
Korrektur & 0,08 & 0,01 & 0,04 & 0,01 & $\mathbf{0 , 7 7}$ & 0,02 & 0,01 \\
Lauter & 0,02 & 0,01 & 0,01 & 0,00 & 0,02 & $\mathbf{0 , 8 6}$ & 0,05 & 0,05 \\
Leiser & 0,04 & 0,04 & 0,01 & 0,00 & 0,02 & 0,02 & $\mathbf{0 , 6 8}$ & 0,11 \\
Zurück & 0,04 & 0,01 & 0,08 & 0,00 & 0,06 & 0,06 & 0,08 & $\mathbf{0 , 5 8}$ \\
\hline
\end{tabular}


vermutlich noch höher ausfallen (Azadi und Jones 2014). Diese Vermutung bestätigt sich bei Luzhnica et al. (2016), deren Versuchsteilnehmer nach einer drei- bis fünfstündigen Trainingsphase Erkennungsraten von $90 \%$ und mehr erzielen konnten.

In einem nächsten Schritt gilt es, die Erkenntnisse dieser Laborstudie unter realen Bedingungen im Feld zu überprüfen und das entworfene Tacton-Set in konkreten Anwendungsszenarien (z.B. Bedienung eines Fahrkartenautomaten bei Blendung und lauten Umgebungsgeräuschen, Bedienung des Infotainment-Systems im Auto) zu evaluieren, um zu untersuchen, ob hierbei vergleichbare Erkennungsraten erzielt werden und inwieweit diese einen Mehrwert für den Nutzer generieren. Zudem wäre es interessant, das Tacton-Set auch bei Endgeräten zu evaluieren, die von den Nutzern in der Hand gehalten werden (wie Smartphone und Tablet-PC), um herauszufinden, ob sich die Wahrnehmung der Tactons am interagierenden Finger und der haltenden Hand gegenseitig beeinflussen.

\section{Fazit}

Insgesamt erscheint das gewählte Vorgehen bei der Entwicklung des Tacton-Sets sinnvoll. Sowohl existierende Erkenntnisse aus der Literatur als auch die Ideensammlung aus der Gruppenbefragung zur konkreten Ausgestaltung der Tactons wurden bei der Gestaltung des Tacton-Sets berücksichtigt und ermöglichten eine gewisse Einschränkung der unzähligen Möglichkeiten, wie solche Tactons gestaltet werden könnten.

Die Ergebnisse dieses Versuchs zeigen, dass das eingangs vorgestellte Tacton-Set durch eine systematische Überarbeitung nochmals verbessert werden konnte. Sowohl die Gesamterkennungsrate als auch die Erkennungsraten der einzelnen Versuchsbedingungen konnten gesteigert werden.

Nach wie vor sollte die Codierung einzelner Funktionen (v. a. Leiser und Zurück) weiter verbessert werden, um bei allen Tactons geeignete Erkennungsraten von über $80 \%$ zu erreichen, wobei das hier gewählte Vorgehen dazu gut geeignet scheint. Die am schlechtesten erkannten Tactons sollten hierfür bezüglich ihrer Ausgestaltung nochmals genauestens betrachtet sowie deren Verwechslungen mit anderen Tactons analysiert werden.

So kann ein solches Tacton-Set für Standardfunktionen in einem iterativen Prozess unter Einbezug potenzieller Nutzer weiter verbessert werden und einen Beitrag für eine einfachere und intuitive Interaktion mit einem Touchscreen liefern. Die vorliegende Ausarbeitung zeigt somit, wie existierende wissenschaftliche Ergebnisse in einem praktischen Szenario angewendet werden können, und liefert gleichzeitig ein übertragbares Vorgehen für die iterative Gestaltung von Tactons, das für andere Anwendungsfälle genutzt werden kann.

Danksagung Die Autoren danken Frau Gesina Glodek, Herrn Maximilian Eysert und Herrn Chris Plitzer für die Unterstützung bei der Versuchsdurchführung.

Funding Open Access funding provided by Projekt DEAL.

Open Access Dieser Artikel wird unter der Creative Commons Namensnennung 4.0 International Lizenz veröffentlicht, welche die Nutzung, Vervielfältigung, Bearbeitung, Verbreitung und Wiedergabe in jeglichem Medium und Format erlaubt, sofern Sie den/die ursprünglichen Autor(en) und die Quelle ordnungsgemäß nennen, einen Link zur Creative Commons Lizenz beifügen und angeben, ob Änderungen vorgenommen wurden.

Die in diesem Artikel enthaltenen Bilder und sonstiges Drittmaterial unterliegen ebenfalls der genannten Creative Commons Lizenz, sofern sich aus der Abbildungslegende nichts anderes ergibt. Sofern das betreffende Material nicht unter der genannten Creative Commons Lizenz steht und die betreffende Handlung nicht nach gesetzlichen Vorschriften erlaubt ist, ist für die oben aufgeführten Weiterverwendungen des Materials die Einwilligung des jeweiligen Rechteinhabers einzuholen.

Weitere Details zur Lizenz entnehmen Sie bitte der Lizenzinformation auf http://creativecommons.org/licenses/by/4.0/deed.de.

\section{Literatur}

Azadi M, Jones LA (2014) Evaluating vibrotactile dimensions for the design of tactons. IEEE Transaction of Haptics 7(1):14-23

Barber D, Beck C (2015) Effects of Tacton names and learnability. In: Yamamoto S (Hrsg) Human interface and the management of information. Information and knowledge design. Lecture Notes in Computer Science. Springer, Cham, S 335-344

Behrends JC, Bischofberger J, Ehmke H, Frings S, Grissmer S, Hoth M, Kurtz A, Leipziger J, Müller F, Pedain C, Rettig J, Wagner C, Wischmeyer E, Deutzmann R (2012) Physiologie, 2. Aufl. Thieme, Stuttgart

Brewster SA, Brown LM (2004) Tactons: structured tactile messages for non-visual information display. In: Cockburn A (Hrsg) Proceedings of the fifth conference on Australasian user interface (AUIC'04), S 15-23

Brewster SA, King A (2005) An investigation into the use of Tactons to present progress information. In: Costabile MF, Paternó F (Hrsg) Human-Computer-Interaction-INTERACT 2005. Springer, Berlin Heidelberg, S 6-17

Brown LM, Brewster SA, Purchase HC (2005) A first investigation into the effectiveness of tactons. In: First joint Eurohaptics conference and symposium on haptic interfaces for virtual environment and teleoperator systems, S 167-176

Brown LM, Brewster SA, Purchase HC (2006) Multidimensional tactons for non-visual information presentation in mobile devices. In: Proceedings of the 8th conference on Human-computer interaction with mobile devices and services (Mobile HCI'06), S 231-238

Chan A, MacLean K, McGrenere J (2005) Learning and identifying haptic icons under workload. In: First joint Eurohaptics conference and symposium on haptic interfaces for virtual environment and teleoperator systems, S 432-439

Chan A, MacLean K, McGrenere J (2008) Designing haptic icons to support collaborative turn-taking. Int $\mathbf{J}$ Hum Comput Stud 66(5):333-355

Constantin A, Brewster SA (2010) Tactile feedback for ambient awareness in mobile interactions. In: Proceedings of the 24th BCS interaction specialist group conference (BCS '10), S 412-417 
van Erp JBF (2002) Guidelines for the use of Vibro-tactile displays in human computer interaction. In: Eurohaptics 2002-Conference Proceedings, S 18-22

Ferguson J, Williamson J, Brewster S (2018) Evaluating mapping designs for conveying data through tactons. In: Proceedings of the 10th nordic conference on human-computer-interaction ACM, New York, S 215-223

Franke H (2016) Problemlösen in Gruppen. Veränderungen im Unternehmen zielwirksam realisieren, 3. Aufl. Edition Rosenberger. Springer, Wiesbaden

Freeman E, Wilson G, Vo DB, Ng A, Politis I, Brewster S (2017) Multimodal feedback in HCI: haptics, non-speech audio, and their applications. In: Oviatt S, Schuller B, Cohen P, Sonntag D, Potamianos G, Krüger A (Hrsg) The handbook of multimodal-multisensor interface. Association for Computing Machinery and Morgan \& Claypool, San Rafael, S 277-317

Gescheider GA, Bolanowski SJ, Verrillo RT (2004) Some characteristics of tactile channels. Behav Brain Res 148(1-2):35-40

Jones LA, Singhal A (2018) Perceptual dimensions of vibrotactile actuators. In: 2018 IEEE haptics symposium IEEE, S 307-312

Karrer K, Glaser C, Clemens C, Bruder C (2009) Technikaffinität erfassen: der Fragebogen TA-EG. In: Lichtenstein A (Hrsg) Der Mensch im Mittelpunkt technischer Systeme 8. Berliner Werkstatt Mensch-Maschine-Systeme (Berlin 2009). Fortschritt-Berichte/VDI: Reihe 22, Mensch-Maschine-Systeme 29, S 194-199

Luzhnica G, Veas E (2019) Background Perception and Comprehension of Symbols Conveyed through Vibrotactile Wearable Displays. In: 24th International Conference on Intelligent User Interfaces (IUI 2019) ACM, S 57-64

Luzhnica G, Veas E, Pammer V (2016) Skin reading: encoding text in a 6-channel haptic display. In: Proceedings oft he 2016 ACM International Symposium on Wearable Computers ACM, S 148-155

MacLean KE, Schneider OS, Seifi H (2017) Multisensory haptic interactions: understanding the sense and designing for it. In: Oviatt $S$,
Schuller B, Cohen P, Sonntag D, Potamianos G, Krüger A (Hrsg) The handbook of multimodal-multisensor interface. Association for Computing Machinery and Morgan \& Claypool, San Rafael, CA, S 97-142

Meuser M, Nagel U (2009) Das Experteninterview - konzeptionelle Grundlagen und methodische Anlage. In: Pickel S, Pickel G, Lauth HJ, Jahn D (Hrsg) Methoden der vergleichenden Politikund Sozialwissenschaft. VS, Wiesbaden, S 465-479

Oldfield RC (1971) The assessment and analysis of handedness: The Edinburgh inventory. Neuropsychologia 9(1):97-113

Pescara E, Fillipov I, Beigl M (2019) Stocksense-A wrist-worn vibrotactile display for tracking volatile markets. In: Proceedings oft he 10th Augmented Human International Conference 2019 ACM, S Art.No.4

Schmid R (1992) Produktionsanlagen perfekt planen und beschaffen. Praxisorientierte Methoden und Techniken, untermauert mit einem Beispiel. Expert, Ehningen bei Böblingen

Stein T, Seeger M, Borys BB, Schmidt L (2017) Design recommendations for Tactons in touch screen interaction. IADIS Int J WWW Internet 15(2):62-77

Stein T, Seeger M, Schmidt L (2016) Die vibrotaktile Wahrnehmung des Menschen an einer ebenen Oberfläche, Arbeit in komplexen Systemen. In: Gesellschaft für Arbeitswissenschaft (Hrsg) Digital, vernetzt, human?! 62. Kongress der Gesellschaft für Arbeitswissenschaft, Aachen, S 1-6 (A.4.24)

Ullrich P (2006) Das explorative ExpertInneninterview: Modifikationen und konkrete Umsetzung der Auswertung von ExpertInneninterviews nach Meuser/Nagel. In: Engartner T, Kuring D, Teubl T (Hrsg) Die Transformation des Politischen. Analysen, Deutungen und Perspektiven; siebentes und achtes DoktorandInnenseminar der Rosa-Luxemburg-Stiftung, S 100-109

Verrillo RT (1992) Vibration sensation in humans. Music Percept Interdiscip J 9(3):281-302 